\title{
Regional patterns of atrophy on MRI in Alzheimer's disease: Neuropsychological features and progression rates in the ADNI cohort ${ }^{*}$
}

\author{
Ranjan Duara, ${ }^{1,2,4 \#}$, David A. Loewenstein ${ }^{1,3}$, Qian Shen ${ }^{1,2}$, Warren Barker $^{1}$, Maria T. Greig ${ }^{1}$, \\ Daniel Varon ${ }^{1,4}$, Melissa E. Murray ${ }^{5}$, Dennis W. Dickson ${ }^{5}$

\footnotetext{
${ }^{1}$ Wien Center for Alzheimer's Disease and Memory Disorders, Mount Sinai Medical Center, Miami Beach, USA;

\#Corresponding Author: duara@msmc.com

${ }^{2}$ Department of Neurology, Miller School of Medicine, University of Miami, Miami, USA

${ }^{3}$ Department of Psychiatry, University of Miami School of Medicine, Miami, USA

${ }^{4}$ Herbert Wertheim College of Medicine, Florida International University, Miami, USA

${ }^{5}$ Mayo Clinic, Jacksonville, USA
} \\ Received 26 August 2013; revised 30 September 2013; accepted 7 October 2013 \\ Copyright (C) 2013 Ranjan Duara et al. This is an open access article distributed under the Creative Commons Attribution License, \\ which permits unrestricted use, distribution, and reproduction in any medium, provided the original work is properly cited.
}

\section{ABSTRACT}

Background: Discrete clinical and pathological subtypes of Alzheimer's disease (AD) with variable presentations and rates of progression are well known. These subtypes may have specific patterns of regional brain atrophy, which are identifiable on MRI scans. Methods: To examine distinct regions which had distinct underlying patterns of cortical atrophy, factor analytic techniques applied to structural MRI volumetric data from cognitively normal $(C N)(n=202)$, amnestic mild cognitive impairment $(\mathrm{aMCl})(n=333)$ or mild $A D(n=146)$ subjects, in the Alzheimer's Disease Neuroimaging Initiative (ADNI) database was applied. This revealed the existence of two neocortical (NeoC-1 and NeoC-2), and a limbic cluster of atrophic brain regions. The frequency and clinical correlates of these regional patterns of atrophy were evaluated among the three diagnostic groups, and the rates of progression from aMCI to $A D$, over 24 months were evaluated. Results: Discernable patterns of regional atrophy were observed in about $29 \%$ of $\mathrm{CN}, 55 \%$ of aMCl and $83 \%$ of $A D$ subjects. Heterogeneity in clinical presentation and $A P O E \& 4$ frequency were associated with regional patterns of atrophy on MRI scans. The most rapid progression rates to dementia among aMCl subjects $(n=224)$,

*Disclosure: The authors report no conflicts of interest.

Statistical analysis performed by David Loewenstein, PhD. over a 24-month period, were in those with NeoC-1 regional impairment $(68.2 \%)$, followed by the Limbic regional impairment $(48.8 \%)$. The same pattern of results was observed when only aMCI amyloid positive subjects were examined. Conclusions: The neuroimaging results closely parallel findings described recently among $A D$ patients with the hippocampal sparing and limbic subtypes of AD neuropathology at autopsy. We conclude that NeoC-1, Limbic and other patterns of MRI atrophy may be useful markers for predicting the rate of progression of $\mathrm{aMCl}$ to $A D$ and could have utility selecting individuals at higher risk for progression in clinical trials.

Keywords: Subtypes; Mild Cognitive Impairment; $\mathrm{MCl}$; preMCl; Amnestic MCl; Alzheimer's Disease; Dementia; MRI; Hippocampal Volume; Algorithmic Diagnosis; Clinical Diagnosis; Neuropsychological Tests; Longitudinal Analysis; Regional Atrophy

\section{INTRODUCTION}

The earliest clinical manifestations of Alzheimer's disease (AD) occur well before the emergence of a dementia syndrome and progression typically occurs at a gradual pace. However, there is considerable variability in clinical presentations and in progression rates [1], which may be attributable to biological subtypes of $A D$, as well as to the presence of comorbid conditions, methodological limitations in the tests used to assess the fea- 
tures and severity of the disease and the disparate responses of subjects to these tests. Heterogeneity in progression rates in $\mathrm{AD}$ may reflect biological variability or errors in the measurement of cognitive and functional status, due to factors such as cognitive reserve, anxiety, motivation and informant bias.

A recent pathological study of $\mathrm{AD}$ cases has shown that the regional distribution of neurofibrillary pathology in the brain appears to be the biological basis for some of this heterogeneity, including variable presentations and progression rates [2]. In this study, pathological subtypes of $\mathrm{AD}$ included a limbic predominant subtype, with older age of onset, female predominance and slow rate of progression, and a neocortical (NeoC) predominant subtype, with younger age of onset, male predominance and a more rapid rate of progression. Given that these neuropathologic subtypes of $\mathrm{AD}$ were identified on the basis of the regional distributions of neurofibrillary pathology, usually in very advanced stages of the disease, it seems reasonable to assume that other pathologic subtypes of the disease, with individual rates of progression, may exist among living subjects with earlier stages of the disease.

Structural MRI has been shown to be one of the best and most readily available measures to assess the biological heterogeneity and rate of progression of $\mathrm{AD}$ [3]. MRI, which has been used to evaluate the rates of atrophy in the hippocampus and the whole brain as well as increases in ventricular volumes, has been found to be reliable and accurate index of the rate of progression of $\mathrm{AD}$, correlating with cognitive and functional measures of progression and potentially, of the response to disease-modifying treatments in clinical trials [4-6]. Because MRI measures of regional cerebral atrophy in AD brains are highly correlated to the severity of regional neurofibrillary pathology, which in turn is correlated to severity of cognitive impairment, structural MRI may be the best method to identify both pathological and clinical subtypes of $\mathrm{AD}$ among living subjects.

While the prevailing emphasis has been to use MRIbased measures, such as whole brain, hippocampal atrophy and ventricular enlargement, to assess disease severity and to predict and measure rates of progression, these traditional measures do not fully capture the heterogeneity of atrophy patterns of the disease at baseline, [4]. Therefore, we performed a factor analysis on volumetric data from subjects participating in the Alzheimer's Disease Neuroimaging Initiative (ADNI), so as to identify distinct patterns of regional atrophy on baseline structural MRI. We then stratified subjects into unique subgroupings based upon the patterns of atrophy present in each individual, thereby enabling us to examine the association of the presence of one or more regional patterns of atrophy to demographic, clinical and neuropsycholo- gical presentations and progression rates in each subject.

\section{METHODS}

Data used in the preparation of this article were obtained from the Alzheimer's Disease Neuroimaging Initiative (ADNI) database (adni.loni.usc.edu). The ADNI was launched in 2003 by the National Institute on Aging (NIA), the National Institute of Biomedical Imaging and Bioengineering (NIBIB), the Food and Drug Administration (FDA), private pharmaceutical companies and nonprofit organizations, as a $\$ 60$ million, 5-year public-private partnership. The primary goal of ADNI has been to test whether serial magnetic resonance imaging (MRI), positron emission tomography (PET), other biological markers, and clinical and neuropsychological assessment can be combined to measure the progression of mild cognitive impairment (MCI) and early Alzheimer's disease (AD). Determination of sensitive and specific markers of very early $\mathrm{AD}$ progression is intended to aid researchers and clinicians to develop new treatments and monitor their effectiveness, as well as lessen the time and cost of clinical trials.

The Principal Investigator of this initiative is Michael W. Weiner, MD, VA Medical Center and University of California-San Francisco. ADNI is the result of efforts of many co-investigators from a broad range of academic institutions and private corporations, and subjects have been recruited from over 50 sites across the US and Canada. The initial goal of ADNI was to recruit 800 subjects but ADNI has been followed by ADNI-GO and ADNI-2. To date these three protocols have recruited over 1500 adults, ages 55 to 90 , to participate in the research, consisting of cognitively normal older individuals, people with early or late MCI, and people with early AD. The follow up duration of each group is specified in the protocols for ADNI-1, ADNI-2 and ADNI-GO. Subjects originally recruited for ADNI-1 and ADNI-GO had the option to be followed in ADNI-2. For up-to-date information, see www.adni-info.org.

In 2011, we downloaded from the ADNI database baseline demographic, clinical, neuropsychological, APOE genotype and volumetric MRI data for 681 subjects [146 diagnosed with mild Alzheimer's disease (AD), 333 with amnestic MCI (aMCI), and 202 cognitively normal (CN) cases]. MRI volumetric scans were performed using 1.5 $\mathrm{T}$ and 3.0 T Siemens, General Electric or Philips scanners. Regional MRI volumes, normalized to total intracranial volume, were obtained from the ADNI database using data derived by researchers at the University of California, San Francisco who used FreeSurfer (FS) version 4.3.0.

MRI data analysis for this study was done in two 
phases, namely among the entire cohort described above (Phase 1), and among only those aMCI and clinically diagnosed $\mathrm{AD}$ subjects who were amyloid positive (Phase 2), using established standardized uptake value ratios (SUVR) cut points (i.e., SUVR $=1.4+$ for scans using [C-11] Pittsburgh Compound B, and 1.11+ for scans using [F-18] Florbetapir).

We used regional brain volumes for 80 available supratentorial brain regions among the 333 aMCI subjects to identify for further analysis those regional volumes which were 1.0 SD or below the mean volume for the same regions in the $\mathrm{CN}$ group; 1) this resulted in 16 brain regions in the aMCI group which met criteria for being atrophic, including entorhinal cortex, hippocampus, amygdala, parahippocampal gyrus, temporal pole, fusiform gyrus, inferior parietal lobule (excluding the supramarginal gyrus), precuneus, middle and inferior temporal, rostral middle frontal, posterior/isthmus cingulate, transverse temporal, superior temporal, insula and supramarginal gyrus; 2) a factor analysis, using the entire group of 681 subjects (CN, aMCI and mild AD) was conducted, separately for the left and right hemispheres, using a Principal Components Approach (PCA) with a varimax rotation to derive the most orthogonal factors; 3) three factors were derived from this analysis, using the criteria of an Eigen Value of 1.0 or greater after inspection of the scree plot, with each factor representing a unique pattern of intercorrelations among specific brain regions (the total explained variance for the left hemisphere was $55.8 \%$ and for the right hemisphere was $56.2 \%)$; 4) individual brain regions which were correlated with the overall factor with a correlation of 0.4 or greater were considered as loading on that particular factor; 5) this resulted in the following factors being derived from the left and right hemispheres (see Figure 1): a Limbic Factor (entorhinal cortex, hippocampus, amygdala, parahippocampal gyrus, temporal pole, fusiform gyrus), a Neocortical Type 1 (NeoC-1) Factor (inferior parietal, precuneus, middle and inferior temporal and rostral middle frontal) and a Neocortical Type 2 (NeoC2) Factor (transverse temporal, superior temporal, insula, supramarginal gyrus and posterior/isthmus cingulate); 6) factor scores were then calculated for Limbic, NeoC-1, NeoC-2 brain regions for each subject, in the left and right hemispheres, and those factor scores which were 1.0 SD or below the entire sample as a whole were designated as atrophic in a given individual and used to categorize the specific pattern of atrophy present in each individual (No atrophy, Limbic, NeoC-1, NeoC-2, or the following combinations of Limbic, NeoC-1 and NeoC-2, NeoC-1 + NeoC-2, Limbic + NeoC-1/NeoC-2. Factor scores were then entered into predictive equations, as described below.

\subsection{Phase 2}

To increase the likelihood that the derived factors in the entire ADNI sample described in Phase 1 were associated with $\mathrm{AD}$ pathology and not merely artifacts of normal aging or other non-AD neurodegenerative pathology, the following steps were taken: 1) We used regional brain volumes for 80 available supratentorial brain regions in the right and left hemispheres among the 83 aMCI subjects in ADNI who were amyloid positive and
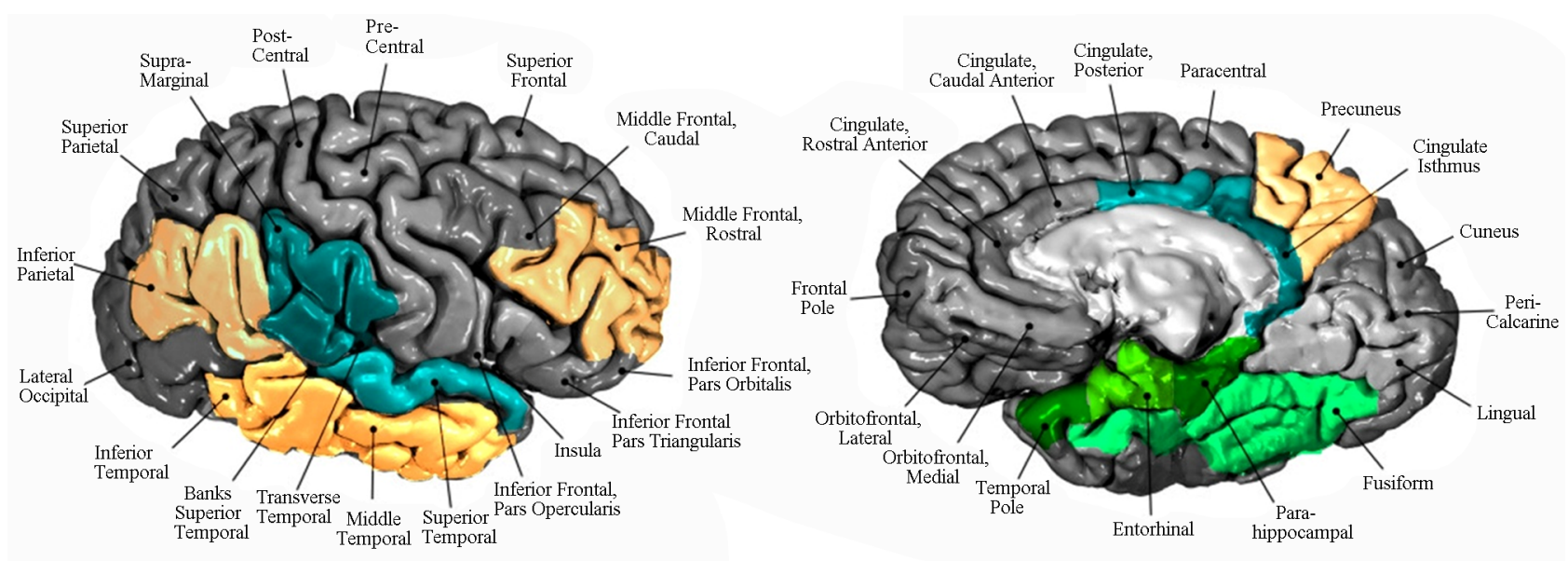

Figure 1. Patterns of regional brain atrophy. Factor analysis of atrophic regions resulted in three subtypes depicted on an atlas created by Desikan et al., 2006 [28]: Limbic pattern depicted in green includes the following regions: Entorhinal cortex, parahippocampal gyrus, temporal pole, fusiform gyrus. Hippocampus and amygdala are part of this pattern of atrophy but are not seen in the figure. Neocortical Type 1 (NeoC-1) pattern depicted in yellow includes the following regions: Inferior parietal, precuneus, middle and inferior temporal and rostral middle frontal. Neocortical Type 2 (NeoC-2) Pattern depicted in blue includes the following regions: Transverse temporal, superior temporal, insula, supramarginal gyrus and posterior/isthmus cingulate. Cortical regions based on Desikan et al., 2006 [28]. 
compared them to 57 normal elderly subjects who were amyloid negative; 2) we then conducted statistical analyses and determined 16 regions among amyloid positive subjects which were atrophic in the left and right hemispheres at $p \leq 0.05$ level of significance. These 16 regions were similar to those identified in Section A and included the entorhinal cortex, hippocampus, amygdala, parahippocampal gyrus, fusiform gyrus, inferior parietal lobule (excluding the supramarginal gyrus), precuneus, middle and inferior temporal gyri, rostral middle frontal gyrus, posterior/isthmus cingulate gyrus, lateral orbital frontal, superior frontal, superior temporal, insula cortex and caudal middle frontal region; 3) a factor analysis, was conducted using 230 subjects who were likely to have AD pathology (amyloid positive subjects diagnosed as aMCI $[\mathrm{n}=85]$ and probable $\mathrm{AD}$, diagnosed using ADNI criteria $[\mathrm{n}=145]$ ); 4) factor analysis was conducted, separately for the left and right hemispheres, using a Principal Components Approach (PCA) with a varimax rotation to derive the most orthogonal factors; 5) three factors were derived from this analysis, using the criteria of an Eigen Value of 1.0 or greater after inspection of the scree plot, with each factor representing a unique pattern of intercorrelations among specific brain regions; 6) three factors were derived from this analysis, using the criteria of an Eigen Value of 1.0 or greater after inspection of the scree plot, with each factor representing a unique pattern of intercorrelations among specific brain regions (the total explained variance for the left hemisphere was $52.7 \%$ and for the right hemisphere was $53.4 \%$ ); 7) individual brain regions were considered representative of that factor if the correlations with the overall factor was 0.4 or greater in both hemispheres; 8) the following factors and associated regions (see Figure 1) were: a Limbic Factor (entorhinal cortex, hippocampus, amygdala, parahippocampal gyrus, fusiform gyrus), a Neocortical Type 1 (NeoC-1) Factor (inferior parietal, precuneus, middle and inferior temporal and rostral middle frontal) and a Neocortical Type 2 (NeoC-2) Factor (superior frontal, superior temporal, insula, lateral orbital frontal and posterior cingulate); 9) among 57 normal elderly subjects in ADNI who were amyloid negative, we examined volumetric values of each region that comprised NeoC-1, NeoC-2 and Limbic areas in both the left and right hemispheres; 10) any volume for a structure that was below that of the lowest value obtained by any of the amyloid negative cognitively normal subjects was then considered abnormal when applied to the entire ADNI sample; 11) because different structures within a left or right hemisphere NeoC-1, NeoC-2 or Limbic region were of different sizes, we considered a region as impaired if there were one or more of the regions comprising that region that were lower than any of the volumes for that region obtained by amyloid negative normal controls; 12) By examining impairment of structures within a region (empirically derived from factor analyses), we were able to identify those regions in which significant atrophy was present and then proceeded to categorize the specific pattern of atrophy present in each individual (No atrophy, Limbic, NeoC-1, NeoC-2, or the following combinations of Limbic, NeoC-1 and NeoC-2, NeoC-1 + NeoC-2, Limbic + NeoC-1/NeoC-2). Subsequently, we added the volumes of all structures in the left and right hemispheres representing Limbic, NeoC-1 and NeoC-2 factors to employ in regression equations investigating predictors of cognitive and functional decline longitudinally.

\subsection{Additional Clinical and MRI Variables}

Because hippocampal volumes and ventricular dilatation have commonly been used as predictors of cognitive decline in longitudinal MRI studies, we included both left and right-sided hippocampal and lateral ventricle values as additional predictors of outcome, as described below. We also examined baseline and follow-up values for the Mini-Mental State Examination (MMSE) [8], Clinical Dementia Rating Scale (CDR) [9] sum of boxes, Immediate and Delayed recall of the Rey Auditory Verbal Learning Test (RAVLT) [10], Delayed Memory for a story passage (i.e., Logical Memory) (LM), Trail Making Test Parts A and B and Category Fluency.

\subsection{Longitudinal Follow-Up}

Of the 681 ADNI subjects evaluated at baseline, 495 (166 mild AD, 224 aMCI and 105 EN subjects) had data available at a 24-month follow-up, including a consensus diagnostic evaluation (regarding "conversion" from $\mathrm{CN}$ to aMCI, or aMCI to AD or another dementia) at the respective ADNI sites. Progression from $\mathrm{CN}$ to aMCI occurred in six $(3.6 \%)$ of subjects and from aMCI to dementia in 88 (39.3\%) of subjects. Reversion from aMCI to "CN" occurred in eight (3.6\%) of subjects, while none of the 98 subjects with $\mathrm{AD}$ had a change in diagnosis.

\subsection{Statistical Analyses}

Chi square $\left(\chi^{2}\right)$ analyses were used to determine the comparative distribution of subjects classified as limbic, NeoC-1, NeoC-2, or a combination of these specific (patterns of atrophy). Chi-square analyses and logistic regression, using MRI derived factor scores, were also employed to determine the extent to which progression from aMCI to dementia could be predicted by different patterns of regional atrophy. To determine the utility of a given MRI factor score at baseline to predict scores on tests of cognitive function at 24-month follow-up, we employed linear regression-based approaches. The baseline (T1) cognitive composite score of interest (e.g., 
Trails B) was entered first into predictive models, followed by simultaneous entry of baseline volumetric scores for the hippocampus alone, ventricular volume, NeoC-1, NeoC-2 and limbic factor scores, with the cognitive composite score at T2 (i.e., Trails B) as an outcome measure. These analyses allowed us to examine the prevalence of different patterns of MRI atrophy among, aMCI and mild AD patients as well as to predict the degree that specific patterns of atrophy or combinations of regional atrophy patterns were associated with disease progression over time.

\section{RESULTS}

\subsection{Phase 1 Analyses}

There were no differences between $\mathrm{CN}$, aMCI or mild $\mathrm{AD}$ groups with regards to age $[(\mathrm{F}(2,678)=1.70 ; \mathrm{p}=$ $0.18]$, but as expected, there were differences with regards to MMSE scores $[(\mathrm{F}(2,678)=522.2 ; \mathrm{p}<0.001]$. $\mathrm{CN}$ subjects had the highest MMSE scores (MMSE = 29.2; $\mathrm{SD}=1.0$ ) followed by the aMCI group (MMSE $=$ 27.1; $\mathrm{SD}=1.8$ ) with the lowest scores being obtained by the AD group $(\mathrm{MMSE}=23.5$; $\mathrm{SD}=1.9)$. There were statistically significant group differences with regards to gender $\left[\left(\chi^{2}(\mathrm{df}=2)=7.61 ; \mathrm{p}<0.031\right]\right.$ with the lowest percentage of females in the aMCI group $(36.6 \%)$ versus the $\mathrm{CN}(45.5 \%)$ and the mild AD group (48.6\%). There was a greater percentage of $A P O E \& 4$ carriers in the $\mathrm{AD}$ $(65.8 \%)$ and aMCI $(53.2 \%)$ versus the $\mathrm{CN}$ groups $(28.2 \%)$ $\left[\left(\chi^{2}(\mathrm{df}=2)=53.56 ; \mathrm{p}<0.001\right]\right.$.

\subsubsection{Frequencies of Different MRI Regional Patterns of Atrophy among Diagnostic Groups}

The distribution of different regional patterns of MRI atrophy (Table 1) was significantly different between diagnostic groups $\left[\chi^{2}(\mathrm{df}=10)=173.64 \mathrm{p}<0.001\right]$, with $83 \%$ of $\mathrm{AD}, 55 \%$ of aMCI and $29 \%$ of $\mathrm{CN}$ subjects showing significant atrophy in NeoC-1, NeoC-2 or limbic regions. The most frequent pattern of atrophy among AD patients was NeoC-1 (24.7\%), followed by Limbic
$(21.2 \%)$ and NeoC-2 (6.2\%); combined patterns of regional atrophy comprised $30.8 \%$, whereas $17.1 \%$ had no atrophy. Among aMCI subjects the most frequent pattern of atrophy was Limbic $(18.0 \%)$, followed by NeoC-2 $(12.6 \%)$ and NeoC-1 (10.5\%); combined patterns of atrophy comprised $13.8 \%$, whereas $45 \%$ had no atrophy. Among CN subjects, NeoC-2 (18.3\%) was the most frequent pattern of atrophy, followed by NeoC-1 (5\%) and Limbic $(3.5 \%)$; combined regional patterns of atrophy comprised $2.0 \%$, whereas $71.3 \%$ had no atrophy.

\subsubsection{Volumes among aMCl and Cognitively Normal Subjects with Different Patterns of MRI Atrophy}

Table 2 shows regional volumes of limbic and nonlimbic structures representing distinct patterns of atrophy among cognitively normal and aMCI subjects in the ADNI subject groups. The table demonstrates the frequency of the various predominant patterns of atrophy among normal and aMCI. These findings demonstrate that: the hippocampus, amygdala and ERC have the most severe atrophy in the Limbic region (as would be expected), whereas the precuneus and the posterior cingulate regions show the most severe atrophy among NeoC1 and NeoC-2 groups. Volumes of the ERC region were relatively greater in the NeoC-1 group than in the normal group (emphasizing that the ERC region, specifically, is likely to be neuropathologically uninvolved in the NeoC1 group).

\subsubsection{Demographic, Clinical, ApoE4 Genotype and Neuropsychological Characteristics of Different Regional Patterns of MRI Atrophy}

The comparative demographic, clinical and cognitive features of different MRI patterns of atrophy were examined for $\mathrm{CN}$, aMCI and AD subjects, contrasted with a control group of $\mathrm{CN}$ subjects with scans showing no atrophy $\left(\mathrm{CN}^{\mathrm{MRI}-}\right)(\mathrm{n}=144)$ (Table 3$)$. There were no statistically significant differences with regards to age $[\mathrm{F}$ $(5,442) ; p=0.056]$, although there was a trend for the

Table 1. Frequencies of different MRI-based regional patterns of atrophy by diagnostic groups.

\begin{tabular}{cccc}
\hline MRI-Based Pattern & CN & aMCI \\
\hline Limbic Only & $3.5 \%(\mathrm{~L}=3.0 \%)(\mathrm{R}=2.0 \%)$ & $18.0 \%(\mathrm{~L}=15.3 \%)(\mathrm{R}=14.1 \%)$ & $21.2 \%(\mathrm{~L}=18.5 \%)(\mathrm{R}=24.0 \%)$ \\
NeoC-1 Only & $5.0 \%(\mathrm{~L}=4.0 \%)(\mathrm{R}=3.0 \%)$ & $10.5 \%(\mathrm{~L}=10.8 \%)(\mathrm{R}=9.3 \%)$ & $24.7 \%(\mathrm{~L}=21.2 \%)(\mathrm{R}=25.3 \%)$ \\
NeoC-2 Only & $18.3 \%(\mathrm{~L}=11.4 \%)(\mathrm{R}=13.4 \%)$ & $12.6 \%(\mathrm{~L}=9.9 \%)(\mathrm{R}=9.6 \%)$ & $6.2 \%(\mathrm{~L}=8.2 \%)(\mathrm{R}=5.5 \%)$ \\
NeoC-1 + NeoC-2 & $1.5 \%(\mathrm{~L}=0.5 \%)(\mathrm{R}=1.0 \%)$ & $6.6 \%(\mathrm{~L}=2.7 \%)(\mathrm{R}=3.3 \%)$ & $7.9 \%(\mathrm{~L}=3.4 \%)(\mathrm{R}=4.1 \%)$ \\
Limbic+ (NeoC-1/NeoC-2) & $0.5 \%(\mathrm{~L}=0 \%)(\mathrm{R}=0 \%)$ & $7.2 \%(\mathrm{~L}=3.3 \%)(\mathrm{R}=2.7 \%)$ & $21.9 \%(\mathrm{~L}=11.0 \%)(\mathrm{R}=11.0 \%)$ \\
Unimpaired & $71.3 \%(\mathrm{~L}=81.2 \%)(\mathrm{R}=80.7 \%)$ & $45.0 \%(\mathrm{~L}=58.0 \%)(\mathrm{R}=48.9 \%)$ & $17.1 \%(\mathrm{~L}=37.7 \%)(\mathrm{R}=30.1 \%)$ \\
\hline
\end{tabular}

Abbreviations: $\mathrm{CN}$ - cognitively normal, aMCI — amnestic mild cognitive impairment, $\mathrm{AD}$-Alzheimer's disease, NeoC - neocortical. $\chi^{2}(\mathrm{df}=10)=173.64 ; \mathrm{p}<$ 0.001 . 
Table 2. Normalized regional volumes in aMCI and cognitively normal subjects with different MRI-based patterns of atrophy.

\begin{tabular}{|c|c|c|c|c|c|c|c|}
\hline MRI-Based Patterns & $\begin{array}{l}\text { MRI Normal } \\
\quad(\mathrm{n}=294)\end{array}$ & $\begin{array}{l}\text { Limbic Only } \\
\quad(n=60)\end{array}$ & $\begin{array}{l}\text { NeoC-1 Only } \\
\quad(n=35)\end{array}$ & $\begin{array}{l}\text { NeoC-2 Only } \\
\quad(n=42)\end{array}$ & $\begin{array}{c}\text { NeoC- } 1+\text { NeoC- } 2 \\
\quad(n=22)\end{array}$ & $\begin{array}{c}\text { Limbic }+ \\
(\text { NeoC-1 and/or } \\
\text { NeoC-2) }(n=24) \\
\end{array}$ & $\begin{array}{l}\text { F-Value } \\
(5,471)\end{array}$ \\
\hline Hippocampal Volume & $\begin{array}{c}0.0043^{\mathrm{a}} \\
(\mathrm{SD}=0.0006)\end{array}$ & $\begin{array}{c}0.0031^{\mathrm{c}} \\
(\mathrm{SD}=0.0004) \\
(-\mathbf{2 8 \%})\end{array}$ & $\begin{array}{c}0.0035^{\mathrm{b}} \\
(\mathrm{SD}=0.0005) \\
(\mathbf{- 1 9 \%})\end{array}$ & $\begin{array}{c}0.0038^{\mathrm{b}} \\
(\mathrm{SD}=0.0005) \\
(\mathbf{- 1 2 \% )}\end{array}$ & $\begin{array}{c}0.0035^{\mathrm{b}} \\
(\mathrm{SD}=0.0005) \\
(\mathbf{- 1 9 \% )}\end{array}$ & $\begin{array}{c}0031^{\mathrm{c}} \\
(\mathrm{SD}=0.0003) \\
(-\mathbf{2 8 \%})\end{array}$ & $69.78^{* * *}$ \\
\hline Amygdala & $\begin{array}{c}0.0014^{\mathrm{a}} \\
(\mathrm{SD}=0.0002)\end{array}$ & $\begin{array}{c}0.0010^{\mathrm{d}} \\
(\mathrm{SD}=0.0002) \\
(\mathbf{- 2 9 \%})\end{array}$ & $\begin{array}{c}0.0012^{\mathrm{c}} \\
(\mathrm{SD}=0.0002) \\
(\mathbf{- 1 4 \% )})\end{array}$ & $\begin{array}{c}0.0013^{\mathrm{ab}} \\
(\mathrm{SD}=0.0002) \\
(-\mathbf{7 \%})\end{array}$ & $\begin{array}{c}0.0012^{\mathrm{bc}} \\
(\mathrm{SD}=0.0002) \\
(\mathbf{- 1 4 \% )}\end{array}$ & $\begin{array}{c}0010^{\mathrm{d}} \\
(\mathrm{SD}=0.0002) \\
(-\mathbf{2 9 \%})\end{array}$ & $58.99^{* * *}$ \\
\hline ERC & $\begin{array}{c}0.0025^{\mathrm{a}} \\
(\mathrm{SD}=0.0004)\end{array}$ & $\begin{array}{c}0.0017^{\mathrm{c}} \\
(\mathrm{SD}=0.0004) \\
(-\mathbf{3 2} \%)\end{array}$ & $\begin{array}{c}0.0035^{\mathrm{b}} \\
(\mathrm{SD}=0.0004) \\
(+\mathbf{4 0 \%})\end{array}$ & $\begin{array}{c}0.0024^{\mathrm{a}} \\
(\mathrm{SD}=0.0004) \\
(\mathbf{- 4 \% )}\end{array}$ & $\begin{array}{c}0.0022^{\mathrm{ab}} \\
(\mathrm{SD}=0.0004) \\
(\mathbf{- 1 2 \% )}\end{array}$ & $\begin{array}{c}0.0015^{\mathrm{c}} \\
(\mathrm{SD}=0.0003) \\
(\mathbf{- 4 0 \% )})\end{array}$ & $62.47^{* * *}$ \\
\hline $\begin{array}{c}\text { Precuneus } \\
\text { (+Inferior Parietal) }\end{array}$ & $\begin{array}{c}0.0103^{\mathrm{a}} \\
(\mathrm{SD}=0.0010)\end{array}$ & $\begin{array}{c}0.0100^{\mathrm{ab}} \\
(\mathrm{SD}=0.0010) \\
(-\mathbf{3 \%} \%)\end{array}$ & $\begin{array}{c}0.0086^{\text {cd }} \\
(\mathrm{SD}=0.0008) \\
(-\mathbf{1 7 \%})\end{array}$ & $\begin{array}{c}0.0097^{\mathrm{b}} \\
(\mathrm{SD}=0.0009) \\
(\mathbf{- 5 \%})\end{array}$ & $\begin{array}{c}0.0081^{\mathrm{d}} \\
(\mathrm{SD}=0.0007) \\
(-\mathbf{2 1 \%})\end{array}$ & $\begin{array}{c}0.0091^{\mathrm{bc}} \\
(\mathrm{SD}=0.0012) \\
(-\mathbf{1 2 \%})\end{array}$ & $43.57^{* * *}$ \\
\hline Posterior Cingulate & $\begin{array}{c}0.0039^{\mathrm{a}} \\
(\mathrm{SD}=0.0004)\end{array}$ & $\begin{array}{c}0.0037^{\mathrm{ab}} \\
(\mathrm{SD}=0.0003) \\
(-\mathbf{5 \%} \%)\end{array}$ & $\begin{array}{c}0.0035^{\text {bc }} \\
(\mathrm{SD}=0.0004) \\
(-\mathbf{1 0 \%})\end{array}$ & $\begin{array}{c}0.0036^{\mathrm{bc}} \\
(\mathrm{SD}=0.0004) \\
(\mathbf{- 8 \% )})\end{array}$ & $\begin{array}{c}0.0032^{\mathrm{d}} \\
(\mathrm{SD}=0.0004) \\
(\mathbf{- 1 8 \% )}\end{array}$ & $\begin{array}{c}0.0034^{\text {cd }} \\
(\mathrm{SD}=0.0005) \\
(-\mathbf{1 3 \%})\end{array}$ & $26.36^{* * *}$ \\
\hline $\begin{array}{l}\text { Middle Frontal } \\
\text { Gyrus }\end{array}$ & $\begin{array}{c}0.0082^{\mathrm{a}} \\
(\mathrm{SD}=0.0008)\end{array}$ & $\begin{array}{c}0.0080 \mathrm{a} \\
(\mathrm{SD}=0.0009) \\
(-\mathbf{2 \%})\end{array}$ & $\begin{array}{c}0.0071^{\text {cd }} \\
(\mathrm{SD}=0.0008) \\
(-\mathbf{1 3 \%})\end{array}$ & $\begin{array}{c}0.0077^{\mathrm{ab}} \\
(\mathrm{SD}=0.0008) \\
(-\mathbf{6 \%})\end{array}$ & $\begin{array}{c}0.0067^{\mathrm{d}} \\
(\mathrm{SD}=0.0009) \\
(-\mathbf{1 8 \%})\end{array}$ & $\begin{array}{c}0.0074^{\text {bc }} \\
(\mathrm{SD}=0.0008) \\
(-\mathbf{1 0 \%})\end{array}$ & $25.29^{* * *}$ \\
\hline $\begin{array}{c}\text { Supramarginal } \\
+ \text { Superior Temporal } \\
\text { Gyrus }\end{array}$ & $\begin{array}{c}0.0062^{\mathrm{a}} \\
(\mathrm{SD}=0.0005)\end{array}$ & $\begin{array}{c}0.0058^{\mathrm{b}} \\
(\mathrm{SD}=0.0005) \\
(-\mathbf{6 \%} \%)\end{array}$ & $\begin{array}{c}0.0054^{\mathrm{c}} \\
(\mathrm{SD}=0.0004) \\
(-\mathbf{1 3 \%})\end{array}$ & $\begin{array}{c}0.0054^{\mathrm{c}} \\
(\mathrm{SD}=0.0004) \\
(-\mathbf{1 3 \%})\end{array}$ & $\begin{array}{c}0.0049^{\mathrm{d}} \\
(\mathrm{SD}=0.0004) \\
(-\mathbf{2 1 \%})\end{array}$ & $\begin{array}{c}0.0052^{\text {cd }} \\
(\mathrm{SD}=0.0004) \\
(-\mathbf{1 6 \%})\end{array}$ & $68.51^{* * *}$ \\
\hline $\begin{array}{c}\text { Transverse } \\
\text { Temporal + Insula }\end{array}$ & $\begin{array}{c}0.0022^{\mathrm{a}} \\
(\mathrm{SD}=0.0002)\end{array}$ & $\begin{array}{c}0.0020^{\mathrm{bc}} \\
(\mathrm{SD}=0.0002 \\
(\mathbf{- 9 \%})\end{array}$ & $\begin{array}{c}0.0020^{6} \\
(\mathrm{SD}=0.0002) \\
(\mathbf{- 9 \%})\end{array}$ & $\begin{array}{c}0.0019^{\mathrm{c}} \\
(\mathrm{SD}=0.0001 \\
(\mathbf{1 4 \%})\end{array}$ & $\begin{array}{c}0.0018^{\mathrm{d}} \\
(\mathrm{SD}=0.0002) \\
(\mathbf{- 1 8 \% )}\end{array}$ & $\begin{array}{c}0.0018^{\text {cd }} \\
(\mathrm{SD}=0.0002) \\
(-\mathbf{1 8 \%} \%)\end{array}$ & $60.51^{* * *}$ \\
\hline
\end{tabular}

Note: Means with different alphabetic superscripts are statistically significant at $\mathrm{p}<0.05$ by the Tukey HSD Procedure; Values in parentheses are Standard Deviations. In Table 2, it is evident that, as compared to those who had no regional atrophy ("MRI Normal"), the Limbic pattern had significant atrophy in the limbic structures (hippocampus, Limbic structures only).

Table 3. Clinical and neuropsychological features in aMCI and AD subjects with various MRI-based patterns of atrophy.

\begin{tabular}{|c|c|c|c|c|c|c|c|c|}
\hline MRI-Based Patterns & $\begin{array}{l}\text { Limbic Only } \\
\quad(n=91)\end{array}$ & $\begin{array}{c}\text { NeoC-1 Only } \\
\quad(n=71)\end{array}$ & $\begin{array}{l}\text { NeoC-2 Only } \\
\quad(n=51)\end{array}$ & $\begin{array}{c}\text { NeoC-1 + NeoC-2 } \\
(n=35)\end{array}$ & $\begin{array}{c}\text { Limbic }+ \\
(\mathrm{NeoC}-1 / \mathrm{NeoC}-2) \\
(\mathrm{n}=56)\end{array}$ & $\begin{array}{l}C N^{M R I-v e} \\
(n=144)\end{array}$ & F or $\chi^{2}$ & p-value \\
\hline Age, Years & $75.99(6.3)$ & $73.86(8.8)$ & $76.02(6.5)$ & $75.40(7.6)$ & $77.57(6.3)$ & $74.51(4.9)$ & 2.18 & 0.056 \\
\hline Gender, \% Females & $46.2 \%$ & $43.7 \%$ & $25.5 \%$ & $37.1 \%$ & $33.9 \%$ & $50.0 \%$ & 11.99 & 0.35 \\
\hline APOE 4+ & $58.2 \%$ & $70.4 \%$ & $39.2 \%$ & $57.1 \%$ & $66.1 \%$ & $25.7 \%$ & 56.84 & $<0.001$ \\
\hline MMSE & $25.76^{\mathrm{bc}}(2.6)$ & $25.21^{\mathrm{cd}}(2.0)$ & $26.39^{\mathrm{b}}(1.9)$ & $25.14^{\text {cd }}(2.6)$ & $24.30^{\text {de }}(2.8)$ & $29.13^{\mathrm{a}}(1.0)$ & 77.16 & $<0.001$ \\
\hline CDR Sum of Boxes & $2.68^{\mathrm{bc}}(1.8)$ & $2.85^{\mathrm{cd}}(1.5)$ & $2.01^{\mathrm{b}}(1.4)$ & $2.60^{\mathrm{bc}}(1.7)$ & $3.46^{\mathrm{d}}(2.0)$ & $0.03^{\mathrm{a}}(0.11)$ & 80.23 & $<0.001$ \\
\hline $\begin{array}{c}\text { WMS } \\
\text { (Passage-Delayed } \\
\text { Recall) }\end{array}$ & $2.41^{\mathrm{cd}}(2.7)$ & $2.28^{\mathrm{cd}}(2.5)$ & $4.20^{\mathrm{b}}(2.6)$ & $3.03^{\mathrm{bc}}(2.3)$ & $1.50^{\mathrm{d}}(2.2)$ & $12.98^{\mathrm{a}}(3.5)$ & 267.74 & $<0.001$ \\
\hline AVLT Total Score & $30.60^{\text {bcd }}(9.6)$ & $28.00^{\mathrm{d}}(10.3)$ & $33.69^{\mathrm{b}}(12.3)$ & $29.83^{\text {bcd }}(9.8)$ & $26.29^{\mathrm{d}}(10.1)$ & $52.12^{\mathrm{a}}(10.7)$ & 91.88 & $<0.001$ \\
\hline AVLT-Delayed Recall & $1.42^{\mathrm{bc}}(2.4)$ & $1.44^{\mathrm{bc}}(2.4)$ & 2. $65^{\mathrm{b}}(3.2)$ & $2.34^{\mathrm{b}}(2.3)$ & $0.84^{\mathrm{c}}(1.8)$ & $7.45^{\mathrm{a}}(3.5)$ & 85.44 & $<0.001$ \\
\hline Trails A & $43.75^{\mathrm{b}}(15.0)$ & $66.31^{\mathrm{cd}}(35.7)$ & $55.22^{\mathrm{bc}}(31.7)$ & $65.86^{\mathrm{cd}}(37.4)$ & $58.04^{\mathrm{cd}}(34.4)$ & $36.25^{\mathrm{a}}(12.5)$ & 19.02 & $<0.001$ \\
\hline Trails B & $137.00^{\mathrm{b}}(70.1)$ & $188.88^{\mathrm{cd}}(85.4)$ & $156.20^{\mathrm{bc}}(78.5)$ & $201.73^{\mathrm{d}}(86.1)$ & $191.56^{\mathrm{cd}}(88.2)$ & $85.24^{\mathrm{a}}(36.8)$ & 36.74 & $<0.001$ \\
\hline Category Fluency & $24.30^{\mathrm{b}}(7.1)$ & $22.55^{\mathrm{b}}(7.1)$ & $25.06^{\mathrm{b}}(7.1)$ & $25.86^{\mathrm{cd}}(9.3)$ & $21.39^{\mathrm{b}}(8.3)$ & $35.12^{\mathrm{a}}(7.9)$ & 48.36 & $<0.001$ \\
\hline
\end{tabular}

All data are presented as mean (standard deviation), unless noted otherwise. Abbreviations: NeoC—neocortical, CN-cognitively normal. Note: Means with different alphabetic superscripts are statistically significant at $\mathrm{p}<0.05$ by the Tukey HSD procedure; Values in parentheses are Standard Deviations.

NeoC-1 subjects to be the youngest group and the Limbic $+(\mathrm{NeoC}-1 / \mathrm{NeoC}-2)$ subjects to be the oldest group. The NeoC-2 subjects had the lowest percentage of females $(25 \%)$, whereas the $\mathrm{CN}^{\mathrm{MRI}-}$ subjects had the highest percentage of females $(50 \%)$. There were also group differences with regards to $A P O E \& 4$ frequency $\left(\left[\chi^{2}(\mathrm{df}=\right.\right.$ $5)=56.84 ; \mathrm{p}<0.001]$ with NeoC-1 (70.4\%) and combined Limbic $+(\mathrm{NeoC}-1 / \mathrm{NeoC}-2)$ subjects $(66.1 \%)$ hav- ing the highest $\varepsilon 4$ frequencies, which were significantly higher than $\mathrm{CN}^{\mathrm{MRI}-}(25.7 \%)$ and NeoC-2 subjects $(39.2 \%)$ who had the lowest $A P O E \& 4$ frequencies.

In Table 3, it can be seen that the control group, namely $\left(\mathrm{CN}^{\mathrm{MRI}-}\right)$ subjects, scored higher on all neuropsychological and functional measures than subjects with any pattern of atrophy including NeoC-2. NeoC-2 subjects generally had the least impairment on all tests 
scores, with the exception of scores on Trails A and B and Category Fluency tests, whereas subjects with a predominant Limbic $+(\mathrm{NeoC}-1 / \mathrm{NeoC}-2)$ pattern generally had the most impaired scores on all tests, with the exception of Trails A and B tests. Subjects with a predominant NeoC-2 pattern had significantly better scores than participants with the NeoC-1 and the Limbic + (NeoC-1/ NeoC-2) regional patterns of atrophy on CDR sum of box scores, MMSE, delayed memory for passages and AVLT total scores. In addition, the NeoC-2 subjects had the least impaired scores on tests of memory (WMS and AVLT, overall cognition (MMSE score) and functional ability (CDR score) relative to NeoC-1, NeoC-1 + NeoC-2 and Limbic $+($ NeoC-1/NeoC-2) subjects.

\subsubsection{Rates of Progression of aMCl to Dementia among Different Subjects with Different MRI Patterns of Atrophy}

The rate of progression to dementia over 24 months was evaluated among aMCI subjects, so as to provide a measurable rate of progression to dementia among subjects with different MRI subtypes of atrophy. At baseline, aMCI subjects exhibited no MRI predominant regional patterns of atrophy in age $[\mathrm{F}(5,218)=1.35 ; \mathrm{p}>0.24]$ or gender $\left[\chi^{2}(\mathrm{df}=5)=5.73 ; \mathrm{p}>0.33\right]$. MMSE scores differed among groups [F $(5,218) ; \mathrm{p}<0.005]$, with the aMCI non-atrophic subjects obtaining higher mean MMSE scores $(\mathrm{M}=27.6 ; \mathrm{SD}=1.6)$ than those with predominant NeoC-1 regional atrophy $(\mathrm{M}=26.2 ; \mathrm{SD}=1.7)$, but among the different MRI groupings there was no significant difference in MMSE scores.

As indicated in Table 4, the rate of progression from aMCI to dementia over 24 months was different according to the type of regional pattern of atrophy $\left[\chi^{2}(\mathrm{df}=5)\right.$ $=21.29 ; \mathrm{p}<0.001]$, with about two thirds of NeoC-1 $(68.2 \%)$ and NeoC-1 + NeoC-2 (64.3\%) subjects, half of Limbic + (NeoC-1/NeoC-2) (50\%) and Limbic (48.8\%), one third of NeoC-2 (34.5\%), and about a quarter $(26.0 \%)$ of the non-atrophic group progressing to dementia.

\subsubsection{Predictors of Progression of aMCI to Dementia among Subjects with Different Predominant MRI Patterns of Atrophy}

We used step-wise logistic regression to predict progression to dementia in 24 months among aMCI subjects, including left and right hemisphere factor scores for Limbic, NeoC-1 and NeoC-2 groupings, right and left hippocampal volumes, as well as ApoE4 status and baseline MMSE score. For the right hemisphere, variables that entered into the model was NeoC-1 factor score $(\mathrm{B}=$ -0.86 ; $\mathrm{SE}=0.18$; Wald $=21.67 ; \mathrm{p}<0.001)$, hippocampal volume $(\mathrm{B}=-8.24 ; \mathrm{SE}=3.1 \mathrm{Wald}=7.31 ; \mathrm{p}<0.016$ and Limbic factor score $(\mathrm{B}=-0.45 ; \mathrm{SE}=0.17$; Wald $=$ $6.96 ; \mathrm{p}<0.008)$, correctly classifying $51.1 \%$ of progressors and $84.3 \%$ of non-progressors, with an overall correct classification rate of $71.2 \%$. For the left hemisphere, variables that entered into the model were left hippocampal volume $(\mathrm{B}=-1445.6 ; \mathrm{SE}=511.8 ; \mathrm{Wald}=7.98$; $\mathrm{p}<0.006)$, NeoC-1 $(\mathrm{B}=-0.47$; $\mathrm{SE}=0.17$; Wald $=7.08$; $\mathrm{p}<0.006$ and baseline MMSE $(\mathrm{B}=-0.193$; $\mathrm{SE}=0.09$; Wald $=7.98 ; \mathrm{p}<0.04)$. These predictors correctly classified $46.6 \%$ of progressors and $78.4 \%$ non-progressors, with an overall correct classification rate of $64.4 \%$.

As indicated in Table 5, baseline MMSE score was the strongest predictor of the MMSE score at the 24-month endpoint. However, Limbic, NeoC-1, NeoC-2 volumes all provided additional and statistically significant added explained variance (14.9\%) in predicting 24-month MMSE scores beyond the baseline MMSE score alone. Similar findings were observed for CDR sum of boxes, total recall score of the AVLT and category fluency test. NeoC-1 and Limbic factor scores predicted Trails B, Delay Memory for Passages (Delayed) and AVLT-Delay scores at the 24-month follow-up even after adjusting for baseline cognitive performance on these measures. After controlling for baseline performance on Trails A, Trails A performance at the 24-month follow-up could be predicted by $\mathrm{NeoC}-1$. The strongest MRI-based subtype predictor of cognitive performance for delayed recall tasks was the Limbic factor score, while the strongest predictor of immediate learning and

Table 4. Progression to dementia among aMCI subjects after 24 months.

\begin{tabular}{ccc}
\hline MRI-Based Pattern & Progressed to Dementia & Did Not Progress to Dementia \\
\hline Limbic Only & $48.8 \%$ & $51.2 \%$ \\
NeoC-1 Only & $68.2 \%$ & $31.8 \%$ \\
NeoC-2 Only & $34.5 \%$ & $65.5 \%$ \\
NeoC-1 + NeoC-2 & $64.3 \%$ & $35.7 \%$ \\
Limbic + (NeoC-1/NeoC-2) & $50.0 \%$ & $50.0 \%$ \\
Non-Atrophic & $26.9 \%$ & $73.1 \%$ \\
\hline
\end{tabular}

Abbreviations: NeoC-neocortical. Note: $\chi^{2}(\mathrm{df}=5)=21.29 ; \mathrm{p}<0.001$. 
Table 5. Predictors of cognitive and functional decline at 24-month follow among aMCI subjects.

\begin{tabular}{|c|c|c|c|c|c|}
\hline $\begin{array}{l}\text { Cognitive/Functional } \\
\text { Score (Time 2) }\end{array}$ & Predictors & Standardized Beta & t-Value & $\mathbf{R}^{2}$ (Baseline Cognitive Score) & $\begin{array}{c}\mathbf{R}^{2} \text { (Baseline Cognitive Score + } \\
\text { MRI Measures) }\end{array}$ \\
\hline \multirow{5}{*}{ MMSE } & MMSE-T1 & 0.342 & $6.05^{* * *}$ & $21.6 \%$ & $36.5 \%$ \\
\hline & Left Ventricle & 0.025 & 0.39 & & \\
\hline & Left Limbic & 0.210 & $3.52^{* *}$ & & \\
\hline & Left NeoC-1 & 0.328 & $5.57^{* * *}$ & & \\
\hline & Left NeoC-2 & 0.205 & $3.43^{* *}$ & & \\
\hline \multirow{5}{*}{ CDR SOB } & CDRSOB T1 & 0.393 & $6.89^{* * *}$ & $22.2 \%$ & $34.3 \%$ \\
\hline & Left Ventricle & -0.003 & 0.05 & & \\
\hline & Left Limbic & -0.236 & $-3.88^{* * *}$ & & \\
\hline & Left NeoC-1 & -0.233 & $-3.93^{* * *}$ & & \\
\hline & Left NeoC-2 & -0.189 & $-3.13^{* *}$ & & \\
\hline \multirow{5}{*}{$\begin{array}{c}\text { Memory for } \\
\text { Passage-Delayed }\end{array}$} & Pass Delay T1 & -0.017 & -0.28 & NA & $9.9 \%$ \\
\hline & Left Ventricle & 0.091 & 1.31 & & \\
\hline & Left Limbic & 0.291 & $4.44^{* * *}$ & & \\
\hline & Left NeoC-1 & 0.241 & $3.91^{* * *}$ & & \\
\hline & Left NeoC-2 & 0.064 & 1.00 & & \\
\hline \multirow{5}{*}{ AVLT-Total Recall } & AVLT-Tot T1 & 0.693 & $18.10^{* * *}$ & $57.3 \%$ & $62.6 \%$ \\
\hline & Left Ventricle & 0.032 & 0.72 & & \\
\hline & Left Limbic & 0.162 & $3.97^{* * *}$ & & \\
\hline & Left NeoC-1 & 0.172 & $4.31^{* * *}$ & & \\
\hline & Left NeoC-2 & 0.133 & $3.23^{* *}$ & & \\
\hline \multirow{5}{*}{ AVLT-DEL } & AVLT-Del T1 & 0.556 & $11.85^{* * *}$ & $41.0 \%$ & $46.4 \%$ \\
\hline & Left Ventricle & 0.070 & 1.31 & & \\
\hline & Left Limbic & 0.224 & $4.44^{* * *}$ & & \\
\hline & Left NeoC-1 & 0.186 & $3.91^{* * *}$ & & \\
\hline & Left NeoC-2 & 0.049 & 1.00 & & \\
\hline \multirow{5}{*}{ Trails A } & Trails A T1 & 0.670 & $15.67^{* * *}$ & $52.7 \%$ & $54.8 \%$ \\
\hline & L Ventricle & -0.003 & 1.31 & & \\
\hline & Left Limbic & -0.052 & -1.20 & & \\
\hline & Left NeoC-1 & -0.163 & $-3.71^{* * *}$ & & \\
\hline & Left NeoC-2 & -0.075 & -1.67 & & \\
\hline \multirow{5}{*}{ Trails B } & Trails B T1 & 0.544 & $11.18^{* * *}$ & $39.4 \%$ & $44.9 \%$ \\
\hline & Left Ventricle & 0.064 & 1.17 & & \\
\hline & Left Limbic & -0.111 & $-2.24^{*}$ & & \\
\hline & Left NeoC-1 & -0.209 & $-4.19^{* * *}$ & & \\
\hline & Left NeoC-2 & -0.044 & -0.87 & & \\
\hline \multirow{5}{*}{ Category Fluency } & Cat Fluency T1 & 0.562 & $12.29^{* * *}$ & $40.1 \%$ & $46.6 \%$ \\
\hline & Left Ventricle & -0.041 & -0.77 & & \\
\hline & Left Limbic & -0.111 & $2.29^{*}$ & & \\
\hline & Left NeoC-1 & 0.194 & $4.09^{* * *}$ & & \\
\hline & Left NeoC-2 & 0.126 & $2.54^{*}$ & & \\
\hline
\end{tabular}

Abbreviations: MMSE $=$ Mini-Mental State Examination CDR SOB $=$ Clinical Dementia Rating Scale Sum of Boxes; AVLT $=$ Rey Auditory Verbal Learning Test. Note: ${ }^{*} \mathrm{p}<0.05 ;{ }^{* *} \mathrm{p}<0.01 ;{ }^{* * *} \mathrm{p}<0.001$. 
non-amnestic measures was NeoC-1 factor score. Ventricular dilatation was not predictive of outcome after other variables were entered into regression models.

\subsection{Phase 2 Analyses}

As indicated in the Methods, we re-analyzed our data so that factor derived regional patterns of atrophy were derived from amyloid positive aMCI and clinically diagnosed AD subjects in the ADNI sample. This approach, in which MRI patterns of atrophy were derived only on subjects thought to have underlying AD pathology, provided factor structures which were very similar to those obtained from the entire ADNI cohort. Subsequently, cut-offs for impairment were determined relative to the performance of normal elderly subjects in the ADNI sample who were amyloid negative. There were statistically significant differences in regional patterns of atrophy among the different diagnostic groups $\left[\chi^{2}(\mathrm{df}=14)=\right.$ $215.21 ; \mathrm{p}<0.001]$. Among aMCI subjects, the limbic subtype of atrophy was most frequent $(13.5 \%)$, followed by combined Limbic $+\mathrm{NeoC}-1+\mathrm{NeoC}-2$ (10.8\%) and combined NeoC-1 + NeoC-2. Other predominant patterns of atrophy were NeoC-1 alone $(6.6 \%)$ and NeoC-2 alone $(4.2 \%)$ and another $10.5 \%$ had other combinations or predominant atrophy. Among AD subjects, the most frequent patterns of atrophy were: Combined Limbic + NeoC-1 + NeoC-2 (27.4\%) followed by limbic alone (17.1\%), NeoC-1 + Limbic (14.4\%), NeoC-1 alone (9.6\%) and other combinations of regional atrophy (14.3\%). Among cognitively normal subjects in the ADNI cohort the most frequent pattern of atrophy was NeoC-2 (6.9\%) while additional subjects $(10.5 \%)$ had other patterns of atrophy.

Similar to the analyses we conducted in Phase 1, in Phase 2 analyses we used simultaneous entry of the total volumes of the factorially derived Limbic, NeoC-1 and NeoC-2 regions in the left hemisphere for amyloid positive aMCI subjects and Probable AD patients in the ADNI sample. Results indicated that after controlling for baseline performance and NeoC-2 and Limbic regional volumes, lower total volumes of NeoC-1 regions were predictive of poorer performance at 24 months on the MMSE, CDR sum of boxes, memory (delayed memory for passages), AVLT immediate recall and AVLT delayed recall, Trails A and B and Category Fluency. After controlling for the entry of other variables, lower scores in the Limbic region were associated with lower memory scores at the 24-month follow-up period. Lower volumes in NeoC-2 region were associated with lower scores on Trails B; the severity of ventricular dilatation was not predictive of cognitive or functional decline.

Taken together, these results among amyloid positive aMCI and clinically diagnosed AD subjects replicate the existence of neocortical and limbic subtypes of $\mathrm{AD}$ found in Phase 1 results, and demonstrate that atrophy in NeoC-1 regions is especially predictive of decline on a broad array of neuropsychological and functional measures at 24 months when adjusting for baseline performance and NeoC-2, Limbic and ventricular volumes.

\section{DISCUSSION}

Using structural MRI data from the ADNI public-access database, and factor analytic and principal component analyses methods, we have identified three distinct patterns of regional cerebral atrophy among subjects diagnosed with AD, aMCI and elderly normals. In the entire cohort of 333 subjects, among those diagnosed with $\mathrm{AD}, 83 \%$ of subjects had one or more of these regional patterns of atrophy (Table 1). Among subjects diagnosed as aMCI, $55 \%$ had one or more regional patterns of atrophy, the most frequent one being Limbic (46\%). Among CN subjects, $29 \%$ had one or more regional pattern of atrophy, the most frequent being NeoC-2.

Among the three core MRI-based patterns of atrophy (NeoC-1, NeoC-2 and Limbic), there were certain distinguishable demographic, genetic and cognitive features, as well as rates of clinical progression (see Tables 3 and 4). Subjects with the NeoC-1 pattern of impairment tended to be younger, about equally likely to be male as female, had the highest $A P O E \& 4$ frequencies, the most severe cognitive (especially, non-amnestic) and functional impairment, as well as the most rapidly progressive course. The regions encompassed by the NeoC-1 region included primarily those structures in the default mode network (DMN) [11], namely the precuneus, inferior parietal cortex, the rostral middle frontal cortex and the lateral temporal cortices, which show abnormal functional connectivity early in the course of AD [12]. Regional hypometabolism on fluorodeoxyglucose PET scans and amyloid deposition on amyloid PET scans also occurs early and most severely in the regions comprising the NeoC-1 and the DMN. There is also evidence that disruption in functional connectivity in these regions is present in asymptomatic $A P O E \& 4$ carriers, antedating measurable amyloid deposition in the brain [13].

Subjects with the limbic patterns of atrophy tended to be older than NeoC-1 subjects, equally likely to be male as female, have $A P O E \& 4$ frequencies and rates of progression to dementia intermediate between subjects with NeoC-1 and NeoC-2 patterns, and to be marginally less impaired on global cognitive, memory and functional measures as NeoC-1 subjects, but significantly less impaired on non-amnestic measures. The more severe memory impairment and the relatively mild non-amnestic impairment relative to NeoC-1 associated with the Limbic subtype of atrophy, may be associated with the greater frequency of this subtype among aMCI subjects. Also, the slower progression rates of these subjects, rela- 
tive to those with NeoC-1 subtype of atrophy may be associated with the higher frequency (and lack of progression to dementia) among aMCI subjects. The regions encompassed by the limbic region (hippocampus, entorhinal cortex, amygdala, fusiform gyrus and anterior temporal pole are closely linked and co-activated with the DMN [11].

Subjects with the NeoC-2 pattern of atrophy also tend to be older than NeoC-1 subjects, are more likely to be male than female, have the lowest $\varepsilon 4$ frequencies, the mildest amnestic and functional impairment, relatively greater nonamnestic impairment than among the other subtypes, and the slowest rate of progression among the three subtypes. The very mild cognitive and functional impairment and slow rates of progression to dementia among NeoC-2 subjects may account for our finding that NeoC-2 is the most frequently identified atrophic pattern among $\mathrm{CN}$ subjects and the least common atrophic pattern among $\mathrm{AD}$ subjects. The regions encompassed by the NeoC-2 pattern of atrophy (superior and transverse temporal, insula, the supramarginal gyrus and posterior and isthmus cingulate regions) are located in the "Temporoparietal Junction Area" [14]. Atrophy in these regions would likely be associated with disruption of a core network of functionally connected regions resulting in non-amnestic cognitive dysfunction [15]. ADNI does not have a separate category of subjects with non-amnestic MCI (naMCI), but our prediction would be that the NeoC-2 subtype of atrophy would be most closely associated with naMCI.

The heterogeneity in patterns of atrophy on MRI scans, observed in the current investigation, corresponds, to some extent, to the subtypes of AD pathology reported recently by Murray et al. [2]. In that study, $25 \%$ of primarily end-stage AD patients who came to autopsy had atypical patterns of distribution of regional neuropathology, including a "limbic predominant" (LP) subtype (accounting for $14 \%$ of $\mathrm{AD}$ ) and a "hippocampal sparing" (HpSp) subtype i.e., a predominantly neocortical (NeoC) subtype, which accounted for $11 \%$ of AD. However, the vast majority of cases $(75 \%)$ had a "typical" distribution of pathology, including a combination or limbic and neocortical neurofibrillary pathology. In this study we also found that combinations of neocortical and limbic subtypes accounted for the most frequent atrophy patterns among aMCI and AD subjects. It would also appear that the heterogeneity in progression rates may be associated with the relative proportion of subjects harboring the NeoC-1 or NeoC-2 patterns of atrophy we have identified. Similar to the NeoC-1 subtype found on antemortem MRI scans, the HpSp (i.e., neocortical) subtype defined pathologically, had faster rates of progression, as well as earlier age-of-onset. The limbic predominant subtype identified at autopsy had a slower rate of pro- gression and later age of onset, which bears some similarity to the MRI-based Limbic subtype we have defined here.

It is highly likely that the pathological subtypes, described in autopsied subjects, typically with end-stage disease, may also be present in much earlier stages of $\mathrm{AD}$, detectable as regional atrophy in MRI scans. Volumetric measurements are highly reliable and accurate markers of regional brain atrophy and have been shown to be highly correlated with regional neurodegenerative pathology, even in the preclinical stage of the disease [7,16-18]. Further, regional cerebral atrophy is known to occur early in Alzheimer's disease (AD), and the pattern of atrophy seen in cortical thickness measures (i.e., the "AD signature") can be detected among elderly, cognitively normal subjects $(\mathrm{CN})$ who later go on to develop mild cognitive impairment (MCI) and/or Alzheimer's disease (AD) [19].

In this study we derived three distinct patterns of atrophy on the entire ADNI cohort based on a factor analysis of 16 brain regions, which were selected because they were shown to have significant atrophy among aMCI subjects in the ADNI cohort. These distinct patterns of atrophy were also observed when the sample was restricted to $\mathrm{AD}$ and aMCI subjects who were amyloid positive on amyloid PET scans. In both cases, a subtype with limbic regional atrophy was identified, as well as two distinct patterns of predominant neocortical involvement. Our findings in this cohort of aMCI and mildly demented $\mathrm{AD}$ subjects show similarity to the pathological findings among end-stage $\mathrm{AD}$ patients in the Murray et al. study (2). There were, of course, differences in the findings between the in-vivo ADNI study and the work of Murray and colleagues, as follows: a) in the Murray et al. study, three neocortical regions and two hippocampal regions (CA-1 and subiculum) were selected, a priori, to define the subtypes, whereas in this MRI-based study, 6 limbic regions and 8 neocortical regions were used to derive the atrophic areas; $b$ ) the subjects in the pathological study were diagnosed to have $\mathrm{AD}$, on the basis of hallmark neuropathologic criteria for this disease, whereas in the MRI study, subjects were diagnosed using clinical criteria, which are likely to be nearly $100 \%$ accurate for a pathological diagnosis among amyloid positive Probable AD subjects and somewhat less accurate among subjects with amyloid positive aMCI subjects; c) subjects in the pathological study generally had end-stage $\mathrm{AD}$ and were very demented by the time they came to autopsy, whereas subjects in the MRI-based study had mild AD, aMCI or were cognitively normal.

While hippocampal volume has been used, in genetic and other studies, as a biomarker for $\mathrm{AD}[7,20]$, the variable clinical presentations and rates of progression in 
$\mathrm{AD}$ are not likely to be predicted by atrophy in structures located solely in the medial temporal lobe [21] among Probable AD subjects and perhaps even more so among subjects with aMCI and naMCI. Hippocampal volumes have been found to be much weaker biomarkers of $\mathrm{AD}$ pathology among subjects with aMCI and especially those with PreMCI, than in those with Probable AD $[7,18,22]$. In fact, the lack of amnestic deficits on neuropsychological evaluation among PreMCI subjects who go on to develop aMCI or AD [18,22], suggests the presence of neocortical rather than limbic pathology, in this early stage of disease. Nevertheless, the regional pattern of atrophy, especially in NeoC-1 areas of the neocortex appears to predict the future course of disease.

In Table 4, it can be seen that most of the variance in the rate of progression among aMCI cases is accounted for by baseline cognitive and functional (CDR-sum of boxes) scores themselves. Although MRI measures predict an additional $14 \%$ to $15 \%$ of this variance over that predicted by MMSE or CDR-SB scores, hippocampal volume is not a predictor, ventricular volume accounts for a very minor proportion of the variance and the main MRI predictors are volumes of left sided NeoC-1 regions. The preceding suggests that in the very early preclinical stages of the disease, before cognitive scores have shown any major decline, these MRI measures may be early predictors of disease course.

The findings in mostly late stage AD cases who had come to autopsy and the current findings on MRI scans in $\mathrm{CN}$, aMCI and mild-moderate $\mathrm{AD}$ cases, together suggest that over the course of the disease, there may be an evolution from a single pattern of atrophy (reflecting localized distribution of neurofibrillary pathology) to a combination of various patterns of atrophy in the later stages of the disease. While it is less than clear that the NeoC-2 pattern of atrophy reflects early AD pathology, as opposed to some other degenerative or age-related atrophy, the data presented in Table 3 suggest that the initial clinical features are related to regional patterns of atrophy detectable early in the disease course. As the disease evolves, the regional pattern of atrophy at any particular stage of disease may allow prediction of the subsequent disease course. Specifically, our data seems to suggest that if a patient presents with predominant NeoC-2 atrophy, the course of the disease is likely to be very slow; however, subsequent development of either NeoC-1 or Limbic pattern of atrophy may signal a change in the course to a more rapid rate of clinical progression.

The limitation of the current study lies in the unique characteristics of subjects who volunteer to participate in ADNI, which is rigorous, with the requirement for longitudinal clinical, neuropsychological and imaging studies, and more recently lumbar puncture. Such subjects may not be representative of the typical patient seen in memory clinics or in a doctor's office. For example, about $25 \%-30 \%$ of $\mathrm{CN}$ elderly subjects in most studies have been shown to be amyloid positive on PET scans, whereas $45 \%$ - $50 \%$ of ADNI subjects, who are cognitively normal, have been found to be amyloid positive on PET scans [23]. The strengths of the current study also lie in its use of the large, multimodal and longitudinal database available in ADNI. This has made it possible to show that regional cortical changes in temporal, parietal and frontal cortex have greater utility as outcome measures in predicting the course of AD than traditional hippocampal and ventricular volumes. While other studies have also provided similar evidence [19,21,24-27], our findings are the first to investigate the possibility of using individual patterns of cortical atrophy for predicting progression rates unique to a particular subject.

The findings in this study, especially with regards to predicting future rates of progression according to regional patterns of predominant atrophy, need to be confirmed and generalized to more representative and typical clinic populations. This may then set the stage for more effective planning of patient care and enable stratification of subjects into relatively homogenous subgroups in AD clinical treatment trials, based on expected rates of progression. Moreover, MRI technology is widely available and provides reliably quantifiable measures, which may be used to provide valuable insights into the pathophysiology of cognitive decline in aging and very early neurodegenerative diseases.

\section{ACKNOWLEDGEMENTS}

Data collection and sharing for this project was funded by the Alzheimer's Disease Neuroimaging Initiative (ADNI) (National Institutes of Health Grant U01 AG024904) and DOD ADNI (Department of Defense award number W81XWH-12-2-0012). ADNI is funded by the National Institute on Aging, the National Institute of Biomedical Imaging and Bioengineering, and through generous contributions from the following: Alzheimer's Association; Alzheimer's Drug Discovery Foundation; BioClinica, Inc.; Biogen Idec Inc.; Bristol-Myers Squibb Company; Eisai Inc.; Elan Pharmaceuticals, Inc.; Eli Lilly and Company; F. Hoffmann-La Roche Ltd and its affiliated company Genentech, Inc.; GE Healthcare; Innogenetics, N.V.; IXICO Ltd.; Janssen Alzheimer Immunotherapy Research \& Development, LLC.; Johnson \& Johnson Pharmaceutical Research \& Development LLC.; Medpace, Inc.; Merck \& Co., Inc.; Meso Scale Diagnostics, LLC.; NeuroRx Research; Novartis Pharmaceuticals Corporation; Pfizer Inc.; Piramal Imaging; Servier; Synarc Inc.; and Takeda Pharmaceutical Company. The Canadian Institutes of Health Research is providing funds to support ADNI clinical sites in Canada. Private sector contributions are facilitated by the Foundation for the National Institutes of Health (www.fnih.org). The grantee organization is the Northern California Institute for Research and Education, and the study is coordinated by the Alzheimer's Disease Cooperative Study at the University of Cali- 
fornia, San Diego. ADNI data are disseminated by the Laboratory for Neuro Imaging at the University of California, Los Angeles.

This work was supported by the State of Florida Alzheimer's Disease Initiative, Department of Elder Affairs, Tallahassee, Florida.

Data used in preparation of this article were obtained from the Alzheimer's Disease Neuroimaging Initiative (ADNI) database (adni.loni.usc.edu). As such, the investigators within the ADNI contributed to the design and implementation of ADNI and/or provided data but did not participate in analysis or writing of this report. A complete listing of ADNI investigators can be found at:

http://adni.loni.usc.edu/wp-content/uploads/how to apply/ADNI_Ackn owledgement List.pdf.

\section{REFERENCES}

[1] Komarova, N.L. and Thalhauser, C.J. (2011) High degree of heterogeneity in Alzheimer's disease progression patterns. PLOS Computational Biology, 7, Article ID: e1002251. http://dx.doi.org/10.1371/journal.pcbi.1002251

[2] Murray, M.E., Graff-Radford, N.R., Ross, O.A., Petersen, R.C., Duara, R. and Dickson, D.W. (2011) Neuropathologically defined subtypes of Alzheimer's disease with distinct clinical characteristics: A retrospective study. The Lancet Neurology, 10, 785-796.

http://dx.doi.org/10.1016/S1474-4422(11)70156-9

[3] Jack Jr., C.R., Shiung, M.M., Gunter, J.L., et al. (2004) Comparison of different MRI brain atrophy rate measures with clinical disease progression in AD. Neurology, 62, 591-600. http://dx.doi.org/10.1212/01.WNL.0000110315.26026.EF

[4] Misra, C., Fan, Y. and Davatzikos, C. (2009) Baseline and longitudinal patterns of brain atrophy in MCI patients, and their use in prediction of short-term conversion to AD: Results from ADNI. Neuroimage, 44, 1415-1422. http://dx.doi.org/10.1016/j.neuroimage.2008.10.031

[5] Evans, M.C., Barnes, J., Nielsen, C., et al. (2010) Volume changes in Alzheimer's disease and mild cognitive impairment: Cognitive associations. European Radiology, 20, 674-682. http://dx.doi.org/10.1007/s00330-009-1581-5

[6] Hua, X., Lee, S., Hibar, D.P., et al. (2010) Mapping Alzheimer's disease progression in 1309 MRI scans: Power estimates for different inter-scan intervals. Neuroimage, 51, 63-75. http://dx.doi.org/10.1016/j.neuroimage.2010.01.104

[7] Frisoni, G.B., Fox, N.C., Jack Jr., C.R., Scheltens, P. and Thompson, P.M. (2010) The clinical use of structural MRI in Alzheimer disease. Nature Reviews Neurology, 6, 67-77. http://dx.doi.org/10.1038/nrneurol.2009.215

[8] Folstein, M.F., Folstein, S.E. and McHugh, P.R. (1975) Mini-mental state. A practical method for grading the cognitive state of patients for the clinician. Journal of Psychiatric Research, 12, 189-198. http://dx.doi.org/10.1016/0022-3956(75)90026-6

[9] Morris, J.C. (1993) The Clinical Dementia Rating (CDR): Current version and scoring rules. Neurology, 43, 24122414. http://dx.doi.org/10.1212/WNL.43.11.2412-a
[10] Rey, A. (1964) L'examen clinique en psychologie. Presses Univeritaires de France, Paris.

[11] Buckner, R.L., Snyder, A.Z., Shannon, B.J., et al. (2005) Molecular, structural, and functional characterization of Alzheimer's disease: Evidence for a relationship between default activity, amyloid, and memory. The Journal of Neuroscience, 25, 7709-7717. http://dx.doi.org/10.1523/JNEUROSCI.2177-05.2005

[12] Sheline, Y.I., Raichle, M.E., Snyder, A.Z., et al. (2010) Amyloid plaques disrupt resting state default mode network connectivity in cognitively normal elderly. Biological Psychiatry, 67, 584-587. http://dx.doi.org/10.1016/j.biopsych.2009.08.024

[13] Sheline, Y.I., Morris, J.C., Snyder, A.Z., et al. (2010) APOE4 allele disrupts resting state fMRI connectivity in the absence of amyloid plaques or decreased CSF Abeta42. The Journal of Neuroscience, 30, 17035-17040. http://dx.doi.org/10.1523/JNEUROSCI.3987-10.2010

[14] Mars, R.B., Sallet, J., Schuffelgen, U., Jbabdi, S., Toni, I. and Rushworth, M.F. (2012) Connectivity-based subdivisions of the human right "temporoparietal junction area": Evidence for different areas participating in different cortical networks. Cerebral Cortex, 22, 1894-1903. http://dx.doi.org/10.1093/cercor/bhr268

[15] Spreng, R.N., Mar, R.A. and Kim, A.S. (2009) The common neural basis of autobiographical memory, prospection, navigation, theory of mind, and the default mode: A quantitative meta-analysis. Journal of Cognitive Neuroscience, 21, 489-510. http://dx.doi.org/10.1162/jocn.2008.21029

[16] Sperling, R.A., Aisen, P.S., Beckett, L.A., et al. (2011) Toward defining the preclinical stages of Alzheimer's disease: Recommendations from the National Institute on Aging-Alzheimer's Association workgroups on diagnostic guidelines for Alzheimer's disease. Alzheimers Dement, 7, 280-292. http://dx.doi.org/10.1016/j.jalz.2011.03.003

[17] Morris, J.C. (2006) Mild cognitive impairment is earlystage Alzheimer disease: Time to revise diagnostic criteria. Archives of Neurology, 63, 15-16. http://dx.doi.org/10.1001/archneur.63.1.15

[18] Duara, R., Loewenstein, D.A., Greig, M.T., et al. (2011) Pre-MCI and MCI: Neuropsychological, clinical, and imaging features and progression rates. The American Journal of Geriatric Psychiatry, 19, 951-960. http://dx.doi.org/10.1097/JGP.0b013e3182107c69

[19] Dickerson, B.C. and Wolk, D.A. (2012) MRI cortical thickness biomarker predicts AD-like CSF and cognitive decline in normal adults. Neurology, 78, 84-90. http://dx.doi.org/10.1212/WNL.0b013e31823efc6c

[20] Saykin, A.J., Shen, L., Foroud, T.M., et al. (2010) Alzheimer's disease neuroimaging initiative biomarkers as quantitative phenotypes: Genetics core aims, progress, and plans. Alzheimer's \& Dementia, 6, 265-273. http://dx.doi.org/10.1016/j.jalz.2010.03.013

[21] Holland, D., Brewer, J.B., Hagler, D.J., Fennema-Notestine, C. and Dale, A.M. (2009) Subregional neuroanatomical change as a biomarker for Alzheimer's disease. Proceedings of the National Academy of Sciences of 
the United States of America, 106, 20954-20959. http://dx.doi.org/10.1073/pnas.0906053106

[22] Loewenstein, D.A., Greig, M.T., Schinka, J.A., et al. (2012) An investigation of PreMCI: Subtypes and longitudinal outcomes. Alzheimer's \& Dementia, 8, 172-179. http://dx.doi.org/10.1016/j.jalz.2011.03.002

[23] Scahill, R.I., Schott, J.M., Stevens, J.M., Rossor, M.N. and Fox, N.C. (2002) Mapping the evolution of regional atrophy in Alzheimer's disease: Unbiased analysis of fluid-registered serial MRI. Proceedings of the National Academy of Sciences of the United States of America, 99, 4703-4707. http://dx.doi.org/10.1073/pnas.052587399

[24] Risacher, S.L., Shen, L., West, J.D., et al. (2010) Longitudinal MRI atrophy biomarkers: Relationship to conversion in the ADNI cohort. Neurobiology of Aging, 31, 1401-1418.

http://dx.doi.org/10.1016/j.neurobiolaging.2010.04.029
[25] Spreen, O. and Strauss, E. (1998) A compendium of neuropsychological tests: Administration, norms, and commentary. 2nd Edition, Oxford University Press, New York.

[26] Jagust, W.J., Landau, S.M., Shaw, L.M., et al. (2009) Relationships between biomarkers in aging and dementia. Neurology, 73, 1193-1199. http://dx.doi.org/10.1212/WNL.0b013e3181bc010c

[27] Desikan, R.S., Cabral, H.J., Settecase, F., et al. (2010) Automated MRI measures predict progression to Alzheimer's disease. Neurobiology of Aging, 31, 1364-1374. http://dx.doi.org/10.1016/j.neurobiolaging.2010.04.023

[28] Desikan, R.S., Segonne, F., Fischl, B., et al. (2006) An automated labeling system for subdividing the human cerebral cortex on MRI scans into gyral based regions of interest. NeuroImage, 31, 968-980.

http://dx.doi.org/10.1016/j.neuroimage.2006.01.021 